\title{
Scabraside D Derived from Sea Cucumber Induces Apoptosis and Inhibits Metastasis via iNOS and STAT-3 Expression in Human Cholangiocarcinoma Xenografts
}

\author{
Kanjana Assawasuparerk ${ }^{1 *}$, Thanakorn Rawangchue ${ }^{2}$, Rassameepen \\ Phonarknguen $^{3}$
}

\begin{abstract}
Scabraside D, a sulfated triterpene glycoside, was extracted from the sea cucumber Holothuria scabra. It shows anti-proliferation in many of cancer cell lines, but the function and mechanisms of action of scabraside $D$ in human cholangiocarcinoma (HuCCA) have not previously determined. In this study, we investigated the activity of scabraside D on HuCCA cell apoptosis, lymphangiogenesis and metastasis in a nude mouse model. Scabraside D induced signs of apoptosis, such as cell shrinkage, nuclear condensation, nuclear fragmentation and DNA fragmentation on TUNEL assays, while effectively decreasing expression of $\mathrm{BCl}-2$ but increasing caspase-3 gene level expression. Immunohistochemistry revealed that scabraside $D$ significantly reduced lymphatic vessel density (LVD). Moreover, scabraside D treatment significantly decreased VEGF-C, MMP-9 and uPA gene expression, which play important roles in the lymphangiogenesis and invasion of cancer cells in metastasis processes. Quantitative real-time PCR showed that scabraside D significantly decreased iNOS and STAT-3 gene expression. This study demonstrated that scabraside D plays a role in activation of HuCCA tumor apoptosis and inhibition of lymphangiogenesis, invasion and metastasis through decreasing BCl-2, MMP-9, uPA and VEGF-C and increasing caspase-3 expression by suppression of iNOS and STAT-3 expression. Therefore, scabraside D could be a promising candidate for cholangiocarcinoma treatment.
\end{abstract}

Keywords: Scabraside D - sea cucumber - cholangiocarcinoma - apoptosis - metastasis - iNOS - STAT-3

Asian Pac J Cancer Prev, 17 (4), 2151-2157

\section{Introduction}

Cholangiocarcinoma (CCA) is a cancer that originates from the bile duct epithelial cells. It is an increasing health problem worldwide (Gores, 2003). The highest incident rate is found in northeast Thailand, where people eat raw freshwater fish infested with liver fluke (Opisthorchis viverrini), the parasitic platyhelminth concerned with CCA (Haswell-Elkins et al., 1994). This cancer is one of the most highly metastatic cancer. It is considered an irremediable cancer owing to lack of competent diagnosis, thus most patients at demonstration have improved advanced disease with increasing rate of invasion and metastasis, leading to an increasing mortality rate.

Metastasis is multistep process that concerns spread of cancer cells from the primary cancer to distant organs. Cancer cells must the surrounding tissue, interpenetrate the blood and lymphatic vessels and generate a new tumor mass at distant organs. When cancer cells metastasize, several proteolytic enzymes conduce to the degradation of extracellular matrix (ECM) and basement membrane (BM)
(Antonina, 2005; Pasco et al., 2005) to form a space for cancer cells to move out of the primary cancer. After that, cancer cell detach from the primary cancer which requires the loss of cell-cell adhesion by cancer cells have secreted protease enzymes. These enzymes including matrix metalloproteinases (MMPs) and serine protease such as urokinase plasminogen activator (uPA). MMPs play a major role in ECM degradation concerned with metastasis of cancer (McCawley and Matrisian, 2000; Westermarck and Kähäri, 1999). Matrix metalloproteinase-9 (MMP9) is a member of the MMP family, has been suggested to play a crucial role in cancer invasion and metastasis (Nabeshima et al., 2002; Sanceau et al., 2003). uPA expression has been revealed to be upregulated in many cancers. The expression of uPA has been associated with invasion and metastasis (Dass et al., 2008). uPA can also stimulate various types of MMPs which, in turn degrade ECM.

Cancer lymphangiogenesis is the main role in the dissemination of cancer cells. The lymphangiogenesis facilitate entry of cancer cells into lymphatic vessel and

${ }^{1}$ Preclinic and Applied Animal Science, ${ }^{2}$ Center for Veterinary Diagnosis, ${ }^{3}$ The Monitoring and Surveillance Center for Zoonotic Disease in Wildlife and Exotic Animals, Faculty of Veterinary Science, Mahidol University, Bangkok, Thailand *For correspondence: kanjana.ass@mahidol.edu 
stimulate cancer metastasis (Hoeben et al., 2004). Vascular endothelial growth factors are known to mainly contribute to promote angiogenesis and lymphangiogenesis in cancer (Xue et al., 2009). Vascular endothelial growth factor-C (VEGF-C) are able to encourage cancer lymphangiogenesis in metastasis process (Harold, 2002).

The expression of iNOS and STAT-3 has been reported in various cancers (Kloz et al., 1998; Kojima et al., 1999; Lechner et al., 2005; Han et al., 2012; Janakiram and Rao, 2012). The iNOS derived nitric oxide (NO) and activate STAT-3 expression are related to pro-tumorigenic function including, increase vascularization to promote cancer growth and metastasis and inhibit apoptosis in cancer. Inhibition of iNOS and STAT-3 expression has been shown to suppress cancer invasion vascularization and metastasis in cancer, whereas to activate apoptosis in cancer cells (Swana et al., 1999; Prakobwong et al., 2011; Janakiram and Rao, 2012).

The sea cucumber Holothuria scabra (H. scabra), which is considered a costly gourmet dish in Asian cuisine, and traditional Chinese medicine uses it in tonics and delicacies. It is widely distributed in the Atlantic and Pacific oceans (Kerr and Chen, 1995). Many species of sea cucumber produce triterpene glycosides as a group of saponin, which are poisonous for other organisms and presumably function as a chemical defense against predation (Caulier et al., 2011; Bahrami et al., 2014). Triterpene glycosides shows several biological activities, including antifungal, cytotoxic, hemolytic, cytostatic and immunomodulatory effects (Kitagawa et al., 1989; Stonix et al., 1999; Chludil et al., 2002). In the present, the anti-cancer activity of triterpene glycosides from sea cucumber has attracted significant attention. Frondoside $\mathrm{A}$ is sulfated triterpene glycosides extracted from sea cucumbers Cucumaria frondosa. It suppressed growth of human pancreatic cancer cells and induced apoptosis via caspase dependent (Attoub et al., 2013). Another two sulfated triterpene glycosides, philinopside A (PA) and philinopside $\mathrm{E}(\mathrm{PE})$, isolated from the sea cucumber Pentacta quadrangularis, were both found to demonstrate anti-angiogenic and anti-cancer effects via receptor tyrosine kinase (RTKs) and VEGF-induced kinase insert domain-containing receptor (KDR) signaling pathway (Tian et al., 2005; Tong et al., 2005). Scabraside D, derived from the sea cucumber H. scabra. It is a type of sulfated triterpene glycoside, which inhibits the proliferation of mouse leukemic cells and various kinds of human cancer cells including lung, gastric, colorectal and breast cancer cells (Han et al., 2012), but its mechanisms was still unknown. However, effect of scabraside D on human cholangiocarcinoma cells has not previously been reported. In this studies, we investigated the effect of scabraside D on human cholangiocarcima (HuCCA) tumor apoptosis, lymphangiogenesis and metastasis in xenograft models. This is the first report exhibiting that scabraside D revealed a significant apoptosis and antimetastatic activity in HuCCA tumor xenograft. Regarding the crucial mechanism, the bioactivity of scabraside D may concern the expression of iNOS and STAT-3, which in turn, decreased the expression of Bcl-2, MMP-9, uPA, VEGF-C and increased the expression of caspase- 3 gene

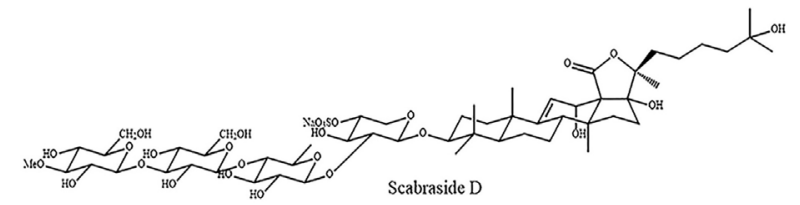

Figure 1. Structure of Scabraside D

level result in apoptotic activation and anti-metastasis in HuCCA tumor xenograft.

\section{Materials and Methods}

\section{Cholangiocarcinoma cell lines}

Human cholangiocarcinoma (HuCCA) cells line CL6 derived from Associate Professor Dr. Adisak Wongkajornsilp, the Department of Pharmacology, Faculty of Medicine Siriraj Hospital, Mahidol University. The cells were cultured in Dulbecco's Modified Eagle Medium (DMEM), containing 15\% fetal bovine serum (FBS) and antibiotic (penicillin $100 \mathrm{IU} / \mathrm{mL}$, streptomycin $(100 \mu \mathrm{g} / \mathrm{mL})$. Cells were maintained at $37{ }^{\circ} \mathrm{C}$ in an incubator with a humidified $95 \%$ and $5 \%(\mathrm{v} / \mathrm{v})$ mixture of air $\mathrm{CO}_{2}$.

\section{Extraction and purification of scabraside $D$}

Scabraside D was extracted from the sea cucumber H. scabra caught from Andaman Sea, Krabi Province, Southern Thailand. Air-dried and finely powdered body walls ( $3 \mathrm{~kg}$, dried weight) of sea cucumber H.scabra were percolated with $2.0 \mathrm{~L}$ of $70 \%$ ethanol for $48 \mathrm{~h}$ twice at room temperature; the ethanol fractions were combined and evapolated in a vacuum chamber to give the crude ethanol extract, which weighed $147.83 \mathrm{~g}$. The extract was dissolved in water and the solution was extracted with n-butanol; the organic fraction was evaporated in a vacuum chamber to yield the butanol fraction, which weighed $6.37 \mathrm{~g}$. The butanol fraction was separated by column chromatography on silica gel (SiO2, $200 \mathrm{~g}$ ), and Sephadex LH-20 column. The purified scabraside D $(10.24 \mathrm{mg})$ in the form of a white power, was validated by $1 \mathrm{H}$ and $13 \mathrm{C}$ NMR spectrum. The chemical structure of scabraside D is shown in Figure 1.

\section{Animal xenograft models}

All mice experiment protocol were approved by the committee for ethics in Faculty of Veterinary Science, Mahidol University, and the experiments were conducted in accordance with the guideline for Animal Experiments of the National Research Council (NRC), Thailand. Sixweek-old female BALB/cMlac-nu mice were purchased from the National Laboratory Animal Center, Salaya District, Nakonpathom Province, Thailand. The. HuCCA cells $(2.5 \times 106$ cells $)$ were suspended in 0.1 of culture medium without FBS and injected subcutaneously into the right flanks of 10 nude mice. The mice were randomly divided into two groups (five animals in each group) and treated with scabraside D ( $1 \mathrm{mg} / \mathrm{kg} / \mathrm{day})$ or vehicle control [100 $\mu$ L normal saline solution (NSS)] by intraperitoneal injections. After 21 days of treatment, all the mice were euthanized at the end of study. Tumor samples were frozen 
for quantitative real-time PCR. Another one was fixed for histopathology and immunohistochemistry with $10 \%$ formalin. Liver and lung of mice were removed and then fixed as same as tumors. Samples fixed with formalin were processed through paraffin embedding.

\section{Histopathology and Metastasis}

In order to investigate histopathology and metastasis. Tumor, liver and lung were stained by routine Hematoxylin and Eosin (H\&E) and to observe nuclear characteristics of tumor xenograft after scabraside D treatment. Nuclear staining was performed using 4'6-diamidino-2phenylindole (DAPI) (Thermo Fisher Scientific, USA) and examined under fluorescent light microscope.

\section{TUNEL assay for apoptotic cells in tumor xenografts}

Apoptosis was evaluated in xenograft tumor using the terminal deoxynucleotidyl transferase mediated dUTPbiotin nick and end labelling (TUNEL) assay, according to the manufacturer's recommendation (Promega, Medison, USA). This assay measured nuclear DNA fragmentation, a significant biochemical indicator of apoptosis (Gavrieli et al., 1992). The extent of apoptosis was assessed by counting the number of TUNEL- positive (brown-stained) cells. Separated by the total number of cells in 10 randomly selected high power fields (magnification, 400x) per tumor (Li et al., 2013).

\section{Immunohistochemistry (IHC)}

For histological analysis of mice xenograft model, tissue section $(5 \mu \mathrm{m})$ were dewaxed and rehydration. The slides were placed in antigen retrieval solution (citrate buffer, $\mathrm{pH}$ 6.0) and heated in a microwave oven for $10 \mathrm{~min}$ at $95{ }^{\circ} \mathrm{C}$. The slides were incubated in a $0.3 \%$ hydrogen peroxidase $(\mathrm{H} 2 \mathrm{O} 2)$ solution for $10 \mathrm{~min}$ to erase endogenous peroxidase. The staining of immunohistochemistry was performed as follows: nonspecific binding was blocked with $10 \%$ normal goat serum. The slides were incubated for $1 \mathrm{~h}$ with primary antibodies, LYVE-1 (lymphatic marker). Primary antibody was as follows: polyclonal rabbit anti-LYVE-1 antibody (1:200 dilution; Millipore Corporation). Next, incubation for $30 \mathrm{~min}$ with a biotin-labeled secondary antibody and color was developed using chromogen peroxidase reagent and counterstained with hematoxylin solution.

\section{Quantification of lymphatic vessel}

Staining quantification was exhibited using light microscopy. The immunostaining sections were scanned at low magnification $(\times 100)$. The areas with the highest amount of positively stained vascular density, called hot spot, were selected for further assessment. The microvessel were counted in a representative high magnification $(x$ 400) (5 fields per tumor). The lymphatic vessel density (LVD) were determined as the mean value of vascular counts. (Straume et al., 2003; Dineen et al., 2008)

\section{Quantitative real-time PCR analysis of gene expression}

Total RNA was isolated from xenograft tumor using TRIzol ${ }^{\circledR}$ Reagent (Invitrogen, Paisley, UK) according to the manufacturer's protocol. Total RNA was converted to
cDNA using the SuperScriptTM III reverse transcription kit following the instruction manual (Invitrogen, Paisley, UK). Quantitative real-time PCR was performed to determine the expression levels of iNOS, STAT-3, uPA, MMP-9, VEGF-C, Bcl-2, caspase-3 and GAPDH. A 20 $\mu \mathrm{L}$ PCR reaction mixture contained $2 \mu \mathrm{L}$ of first-strand cDNA of each primer and $18 \mu \mathrm{L}$ of KAPA SYBR ${ }^{\circ}$ FAST qPCR kit Master Mix (2x) (KAPA Biosystems, Woburn, $\mathrm{MA}$ ), and was processed in an ABI 7500 Real-Time PCR system (Appiled Biosystems, Foster City, CA).

The specific primers were designed according to the full cDNA sequences of iNOS (GeneBank accession no. AF049656.1), STAT-3 (GeneBank accession no. BC001154), uPA (GeneBank accession no. A18395), MMP-9 (GeneBank accession no. NM_004994), VEGF-C (GeneBank accession no.NM_005429), Bcl-2 (GeneBank accession no. BC027258), caspase-3 (GeneBank accession no. NM_004346), and GAPDH (GeneBank accession no. NM_002046) using Primer Blast Program (Ye et al., 2012). The following specific primers (Pacific Science Co., Ltd., Thailand) were used: iNOS, 5'CACTTCCAACGCAACATGGG -3' (sense) and 5'CTTTGACCCAGTAGCTGCCA -3' (antisense) 353 bp product; STAT-3, 5' - CCCCCTACCAAGAAGCACTG-3' (sense) and 5'- ACCGACAGCCAGTCAAAGAG-3' (antisense) 481 bp product; uPA, 5' GGTGTACACTGGATTCGCCA -3' (sense) and 5'TAGACAGATGGGGGTGTCGT-3' (antisense) $281 \mathrm{bp}$ product; MMP-9, 5'- TCGTGGTTCCAACTCGGTTT -3' (sense) and 5'- CGGCCCTCGAAGATGAAGG -3' (antisense) 244 bp product; VEGF-C, 5'-AGGCCAACCTCAACTCAAGG-3' (sense) and 5'TCGCGACTCCAAACTCCTTC-3' (antisense) 156 bp product; Bcl-2, 5'-TGGGAGAACGGGGTACGATA-3' (sense) and 5'-CATGACCCCACCGAACTCAA-3' (antisense) 460 bp product; caspase 3 , 5'-GTTGGCGTCGCCTTGAAATC-3' (sense) and 5'-TGAGGTTTGCTGCATCGACA - 3' (antisense) 349 bp product; and GAPDH, 5'-CTCCTGTTCGACAGTCAGCC-3' (sense) and 5'-TTCCCGTTCTCAGCCTTGAC-3' (antisense) 262 bp product.

The PCR conditions were as follows: the amplification primary incubation at $95^{\circ} \mathrm{C}$ for $10 \mathrm{~min}$, followed by 40 cycles of denaturation at $95^{\circ} \mathrm{C}$ for $10 \mathrm{~min}$, annealing at $60{ }^{\circ} \mathrm{C}$ for $1 \mathrm{~min}$, and extension at $60{ }^{\circ} \mathrm{C}$ for $1 \mathrm{~min}$. The sequences of these PCR products were obtained by direct sequencing. Each amplicon was cloned into PGEM ${ }^{\circledR}-\mathrm{t}$ Vector (Promega, Madison, WI, USA) in order to create standard curves for target cDNA. The mRNA levels were reported as ratios of the copy numbers of target cDNAs to GAPDH cDNA.

\section{Statistical analysis}

Data were expressed as mean \pm SD from three or more independent experiments. Statistical evaluation was performed using significance was accessed by analysis of variance (ANOVA) and student's t-test in GraphPad Prism program version 5 (GgraphPad software, San Diego, CA). Difference with p-values $<0.05$ was considered statistically significant. 


\section{Results}

Scabraside D induces apoptosis of HuCCA tumor xenografts

To evaluate the mode of cell death induced by scabraside D in HuCCA tumor xenografts compared with control group. The untreated control tumor cells displayed large cell, variable shaped nuclei and many dividing cell (Figure 2A, C, E). The scabraside D-treated HuCCA tumor xenografts showed cell shrinkage, nuclear condensation by staining tumor cells with $\mathrm{H} \& \mathrm{E}$ (Figure $2 \mathrm{~B}$ ). The treated tumor cells also showed some degree of nuclear shrinkage and nuclear fragmentation by staining cells with the DNA specific dye, DAPI (Figure 2D). TUNEL staining which is label fragmented DNA. The scabraside D-treated tumor cells displayed dark brown nuclei (Figure 2F). Furthermore, treatment with scabraside D induced a significant increase in the number of TUNEL-positive cells in the tumor cells compared to control (Figure 2G). These morphological variation and evidence of DNA fragmentation on TUNEL assay suggest that apoptotic processes were on-going in HUCCA tumor xenografts treated by scabraside $\mathrm{D}$.

\section{Scabraside D decreased lymphangiogenesis in HuCCA} tumor xenografts

The lymphangiogenesis in the HuCCA tumor was detected by immunohistochemistry. Anti-LYVE-1 was used as a lymphangiogenesis marker and lymphatic vessel density (LVD). LYVE-1 positive vessels lower in scabraside D-treated group than control group. LYVE-1 positive cell clusters were counted as LVD at high power magnification $(\times 400)$. Number of lymphatic vessels/ field was significantly less in scabraside D-treated group $(51.40 \pm 10.11)$ than control group $(81.20 \pm 16.32)$ (Figure 3 ). The results presented the scabraside D prevents lymphangiogenesis in HuCCA tumor.

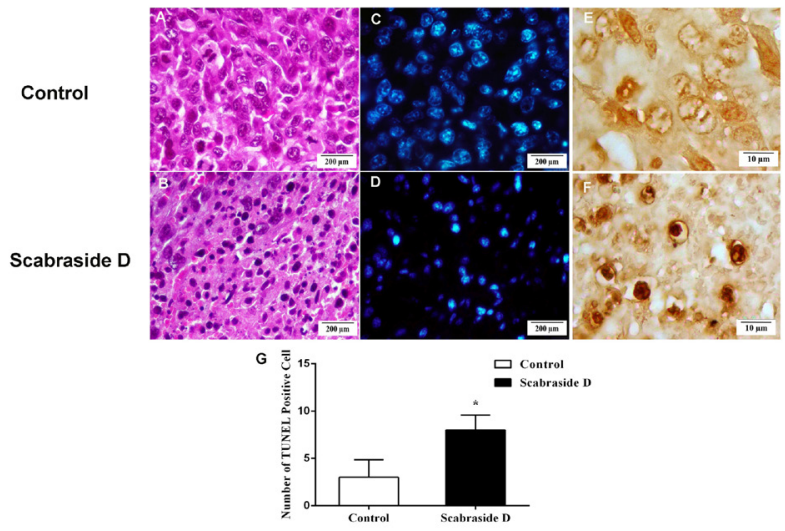

Figure 2. Morphology of HuCCA Tumor Cells in Control (A, C and E) and Scabraside D-treated Group (B, D and F). The scabraside D treated HuCCA cells demonstrated cell shrinkage and nuclear condensation by H\&E staining (B), fragmented nuclei by DAPI staining (D) and dark brown nuclei by TUNEL staining (F). The number of TUNEL positive cells in tumors from scabraside $\mathrm{D}$ treated animals was increased compared with control $(8.00 \pm 1.58$ versus $3.00 \pm 1.87$; 5 tumor per group, 10 high power fields (total magnification; $400 \times$ ) per tumor. The $*$ indicates statistical significance at $\mathrm{p}<$ 0.05 in comparison to control

\section{Scabraside D inhibits metastasis}

Metastasis to liver or lung at 21 days post inoculation was validated by the evaluation of sections stained with H\&E. The sacbraside D treated group, no metastasis was demonstrated in the liver and lung (Figure 4B, 4D). In contrast, the control group showed distant metastasis to liver and lung (Figure 4A, 4C). The liver tissue in the control group showed neoplastic aggregates in liver parenchyma. Besides, the lung tissue in the control group showed mulifocal aggregates of carcinoma cells in lung parenchyma. Meanwhile, the characteristics of liver and lung in the treated group showed no cluster of neoplastic cells in lung and liver parenchyma. This result suggested that scabraside D inhibited HuCCA tumor metastasis.

\section{Effect of scabraside $D$ on gene expression}

The expression level of iNOS, STAT-3, VEGF-C, uPA, MMP, Bcl-2 and Caspase-3 in the scabraside D-treated
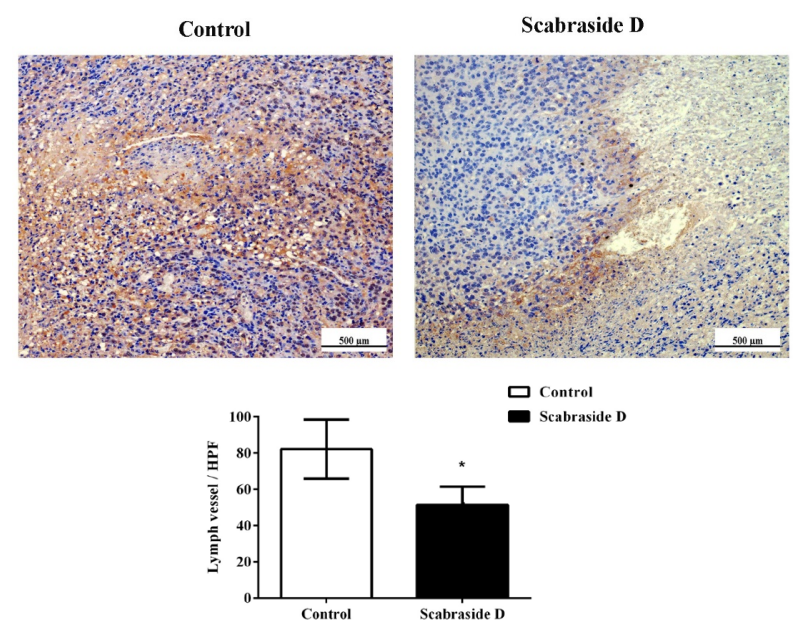

Figure 3. Scabraside D Decreased LVD in HuCCA Tumor Xenografts. HuCCA tumor section from animals treated with scabraside D or control were analyzed for LVD by immunohistochemistry using LYVE-1. The number of lymphatic vessels in tumor from scabraside $\mathrm{D}$ treated nude mice was reduced compared with control $(51.40 \pm 10.11$ versus $81.20 \pm 16.32$; 5 tumor per group, 5 high power fields (total magnification; $400 \times$ ) per tumor. The $*$ indicates statistical significance at $\mathrm{p}<0.05$ in comparison to control

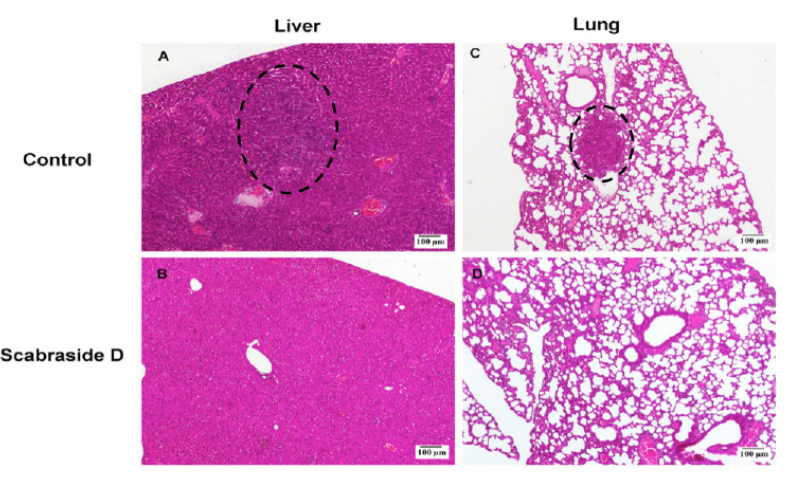

Figure 4. Histopathology by H\&E Staining of Liver $(A, B)$ and Lung $(C, D)$ in Nude Mice at 21 days Post Inoculation. Liver and lung in scabraside $\mathrm{D}$ treated group (B, D) demonstrated normal appearance, but control group (A, C) showed metastatic foci (black dash circle) in liver and lung 

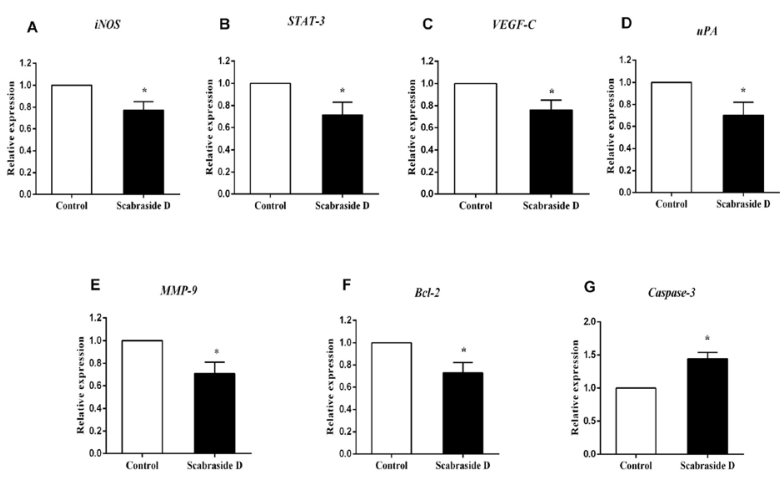

Figure 5. Gene expression results for $\operatorname{iNOS}(\mathrm{A})$, STAT3 (B), VEGF-C (C), uPA (D), MMP-9 (E), Bcl-2 (F) and Caspase-3 (G) in HuCCA tumor cells following scabraside $\mathrm{D}$ treatment. The values are mean $\pm \mathrm{SD}$ relative to the control values, and normalized based on GAPDH $(n=3)$. The $*$ indicates statistical significance at $\mathrm{p}<0.05$ in comparison to control

HuCCA tumor xenografts were determined by quantitative real-time PCR analysis. In comparison to the control group, scabraside D treatment decreased iNOS, STAT-3, VEGF-C, uPA, MMP-9 and Bcl-2 level in HuCCA tumor cells (Figure 5A-F), whereas the expression of caspase-3 increased in the scabraside D-treated group (Figure 5G). In all the values obtained, significant differences to control group $(\mathrm{p}<0.05)$.

\section{Discussion}

Although the incidence and mortality of CCA increasing, usable therapeutics have not significantly extended patient survival. There is need to improve new curative agents that can increase the sensitivity of CCA. The detection of new natural products and their constituents to against cancer has attracted researcher attentions. Marine products contain a diversity of chemotherapeutic compounds that have displayed to preclude cancer development and several marine derived molecules are presently entering clinical trials in cancer treatment (Tian et al., 2005). As previously shown scabraside D extract from sea cucumber $\mathrm{H}$. scabra have anti-proliferation property against five cancer cell lines including, mouse leukemic cells (P-388), lung human cancer cell (A-549), gastric cancer cell (MKN-28), human colorectal cancer cell (HCT-116) and human breast cancer cell (MCF-7) (Han et al., 2012). The effect of scabraside D on HuCCA tumor xenograft has not been reported. Here, we provide evidence that scabraside $\mathrm{D}$, new marine natural sulfated triterpene glycoside is competent of promotion apoptosis and capable of inhibition the major process involve in metastasis, including blocking lymphangiogenesis and suppressing invasion in HuCCA tumor xenograft. This research is the first to reveal that the expression of iNOS and STAT-3 associated with apoptosis, lymphangiogenesis and metastasis in HuCCA tumor xenograft. We found that treated with scabraside D induce apoptosis in HuCCA tumor cells. The effect of scabraside D showed typical characteristic of apoptotic cell, i.e. cell shrinkage, nuclear condensation, nuclear DNA fragmentation. Moreover, we examined the expression of apoptosis-related genes in the scabraside D treated HuCCA tumor cells, we found that the up-regulation of caspase- 3 gene and down-regulation of anti-apoptotic Bcl-2 gene associated with down-regulation of iNOS and STAT-3 expression. Inducible nitric oxide synthase (iNOS) is one of the three significant enzymes generating nitric oxide (NO) (Lechner et al., 2005). The expression of iNOS has been reported in variety of tumors, including prostate carcinoma (Klotz et al., 1998), gynecological carcinoma (Thomsen et al., 1994), colon carcinoma (Kojima et al., 1999) and transitional cell carcinoma of the bladder (Swana et al., 1999). NO expression has many pro-tumorigenic function such as increasing vascularization, which support growth and metastasis, and inhibiting apoptosis (Jakiram and Rao, 2012). The iNOS derived NO can regulate STAT3 expression to promote growth of malignant cancer cells (Carpenter and Lo, 2014). STAT-3 is DNA-binding transcription factor (Akira et al., 1994). STAT-3 is often constitutively stimulated in many human cancer (Aggarwal et al., 2006). Previously, the report found the expression of STAT-3 in CCA cell lines (Prakobwong et al., 2010). STAT-3 activation is also concerned with tumor existence and curative resistance (Carpenter and Lo, 2014). Our data also displayed scabraside D suppressed anti-apoptotic Bcl-2 gene that are regulated iNOS and STAT-3 gene. iNOS and STAT-3 activation have been reported to suppression apoptosis. iNOS expression induce NO-mediated s-nitrosylation of Bcl-2 inhibits it ubiquitination and subsequent proteasomal degradation, result in blocking of apoptosis (Azad et al., 2010). Meanwhile, STAT-3 expressed directly concerned with the $\mathrm{Bcl}-2$ gene promoter and increase $\mathrm{Bcl}-2$ expression thereby inhibiting apoptosis (Choi and Han, 2012). The STAT-3 expression has been revealed to induce expression of inhibitor of caspase (IAPs), such as survivin, which suppress stimulation of caspase to inhibit apoptosis (Gritsko et al., 2006). Our results also showed scabraside $\mathrm{D}$ treatment was associated with downregulation of expression of cell survival gene could be due to observed decrease activation of iNOS and STAT-3. Scabraside D down-regulation of anti-apoptotic Bcl-2 and up-regulation of caspase-3 gene level, suggest that scabraside D induced apoptosis. Hence, scabraside D demonstrate pro-apoptotic activity in HuCCA tumor xenografts, which is concerned with alteration of iNOS/NO/STAT-3 axis. In addition, we found that scabraside $\mathrm{D}$ is competent of inhibition the major step involved in metastasis, together with blocking adhesion, invasion and lymphangiogenesis in HuCCA tumor xenograft model. Our finding showed that scabraside $\mathrm{D}$ had a potent against metastatic activity by inhibition iNOS and STAT-3 gene. One of the major features required for tumor invasion and metastasis is the capability to degrade ECM and BM. This process is completed by the stimulation of variety of proteolytic enzymes, such as MMPs and serine protease which work in concert (Dano et al., 2005). MMPs are crucial enzymes in ECM degradation and metastasis (McCawley and Matrisian, 2000). MMP-9 is major enzyme in cancer metastasis as a result of ECM degradation. uPA, a serine protease that is involved in ECM degradation. Increasing of uPA expression associated lymphatic invasion and 
metastasis in CCA patients (Thummarati et al., 2012). Active uPA cleaves inactive plasminogen to create active plasmin, which is a broad-specific serine protease, can degrade ECM. Plasmin and uPA can stimulate many types of MMPs which in turn EMC degradation (Dano et al., 2005). The iNOS/NO and STAT-3 expression may contribute to the invasive potential of cancer cells by activation proteolytic enzymes, i.e. MMP-9 leading to cancer invasion (Carpenter and Lo, 2014). An inhibitory effect on iNOS and STAT-3 expression is important for blocking proteolytic enzymes, result in anti-invasion and anti-metastasis. From these results demonstrated that treated with scabraside D suppressed MMP-9 and uPA gene level could be owing to observed decrease of activation of iNOS and STAT-3. Beside, we showed $\mathrm{H} \& \mathrm{E}$ staining that treatment with scabraside D had not metastasis in liver and lung. These results suggested that scabraside D inhibit the invasive and metastatic ability of HuCCA tumor xenograft by depressing MMP-9 and uPA expression via downregulation the expression of iNOS and STAT-3. iNOS and STAT-3 are also involved in the regulation of lymphangiogenesis, by which cancer cell promote vascularization for their growth and invasiveness. VEGF-C is the key regulatory factors for cancer lymphangiogenesis, and likely play a major role in the lymphatic cancer metastasis (Yu et al., 2009). Several reports have showed that iNOS and STAT-3 expression correlated with VEGF-C activity in various human cancer cells (Vakkala et al., 2000; Niu et al., 2002; Song et al., 2002; Xu et al., 2002; Storr et al., 2012; Yan et al., 2015; Karadayi et al., 2013). Yan and colleague showed that STAT-3 expression in papillary thyroid cancer was positively related to VEGF-C expression result in enhance LVD and play a major role in the invasive and metastasis (Yan et al., 2015). LVD is positively related to lymphatic vessel invasion and metastasis of the cancer (Yu et al., 2009). Therefore, if the activity of iNOS and STAT-3 are decreased leading to inhibition of VEGF-C expression, and result in blocking cancer lymphangiogenesis and metastasis (Niu et al., 2002; Song et al., 2002; Wang et al., 2005; Karadayi et al., 2013). We also observed that scabraside D treatment markedly suppressed the expression of iNOS, STAT-3, VEGF-C gene levels. Furthermore, treatment with scabraside D reduced LVD compare to control group. Scabraside D was principally to inhibit the expression of iNOS and reduce the STAT-3 level in HuCCA tumor xenograft. These results suggested that scabraside D decreased the lymphangiogenesis ability of HuCCA tumor xenograft, in part through reduced the iNOS and STAT-3 expression.

In conclusion, the recent study provides evidence that scabraside D possesses marked apoptotic and antimetastatic activity. We have demonstrated that scabraside D plays a role in activation of HuCCA tumor apoptosis and inhibition of HuCCA tumor lymphangiogenesis, invasive and metastasis via suppression of Bcl-2, MMP-9, uPA and VEGF-C, and enhancement of caspase- 3 gene level expression by blocking iNOS and STAT-3 expression. To our knowledge, this is the first report demonstrating the apoptotic and anti-metastatic activity of scabraside D to against HuCCA tumor xenografts. These finding identify scabraside D as promising new alternative treatment for cholangiocarcinoma and perhaps other cancer.

\section{Acknowledgements}

We thank Dr. Saksit Nobsathian from the Nakornsawan Campus, Mahidol University, who identified chemical structure of scabraside D. We thank the Monitoring and Surveillance Center for Zoonotic Disease in Wildlife and Exotic Animals, Faculty of Veterinary Science, Mahidol University, for the provision of lab space to this research.

\section{References}

Aggarwal BB, Sethi G, Ahn KS, et al (2006). Targeting signaltransducer-and-activator-of-transcription-3 for prevention and therapy of cancer: modern target but ancient solution. Ann N Y Acad Sci, 1091, 151-69.

Akira S, Nishio Y, Inoue M, et al (1994). Molecular cloning of APRF, a novel IFN-stimulated gene factor 3 p91-related transcription factor involved in the gp 130-mediated signaling pathway. Cell, 77, 63-71.

Antonina H (2005). Progression molecular mechanisms of tumor metastasis and angiogenesis. Anticancer Res, 25, 3327-34.

Attoub S, Arafat K, Gèlaude A, et al (2013). Frondoside A suppressive effects on lung cancer suuvival, tumor growth, angiogenesis, invasion and metastasis. Plos One, 8, 1-10.

Azad N, Iyer AK, Wang L, et al (2010). Nitric oxide-mediated bcl-2 stabilization potentiates malignant transformation of human lung epithelial cells. Am J Respir Cell Mol Biol, 42, 578-85.

Bahrami Y, Zhang W, Chataway T, et al (2014). Structural elucidation of novel saponins in the sea cucumber Holothuria lessoni. Mar Drugs, 12, 4439-73.

Carpenter RL, Lo HW (2014). STAT-3 target genes relevant to human cancers. Cancers, 6, 897-925.

Caulier G, Dyck SV, Gerbaux P, et al (2011). Review of saponin diversity in sea cucumbers belonging to the family Holothuriidae. SPC Beche-de-mer Inf Bull, 31, 48-54.

Chludil HD, Muniain CC, Seldes AM, et al (2002). Cytotoxic and antifungal triterpene glycosides from the Patagonian sea cucumber Hemoiedema spectabilis. J Nat Prod, 65, 860-65.

Choi HJ, Han JS (2012). Overexpression of phospholipase D enhances Bcl-2 expression by activating STAT-3 through independent activation of ERK and p38MAPK in HeLA cells. Biochim Biophys Acta, 1823, 1082-91.

Dano k, Behrendt N, Hoyer-Hansen G, et al (2005). Plasminogen activation and cancer. Thromb Haemost, 93, 676-81.

Dass K, Ahmad A, Azmi AS, et al (2007). Evolving role of uPA/uPAR system in human cancers. CancerTreat Rev, 34, 122-36.

Dineen SP, Sullivan LA, Beck Aw, et al (2008). The adnectin CT-322 is novel VEGF receptor 2 inhibitor that decrease tumor burden in an orthotopic mouse model of pancreatic cancer. BMC Cancer, 8, 1-10.

Gavrieli Y, Sherman Y, Ben-Sasson SA (1992). Identification of programmed cell death in situ via specific labeling of nuclear DNA fragmentation. J Cell. Biol, 119, 493-501.

Gores GJ (2003). Cholangiocarcinoma: current concepts and insights. Hepatol, 37, 961-69.

Gritsko T, Williams A, Turkson J, et al (2006). Persistent activation of STAT-3 signaling induces survivin gene expression and confers resistance to apoptosis in human breast cancer cells. Clin Cancer Res, 12, 11-19.

Han H, Li L, Yi YH, et al (2012). Triterpene glycosides from sea cucumber Holothuria scabra with cytotoxic activity. Chin 
Herb Med, 4, 183-88.

Harold FD (2002). Vascular permeability factor/vascular endothelial growth factor: a critical cytokine in tumor angiogenesis and potential target for diagnosis and therapy. J Clin Oncol, 20, 4368-80.

Haswell-Elkins MR, Mairiang E, Mairiang P, et al (1994). Cross-sectional study of Opisthorchis viverrini infection and cholangiocarcinoma in communities within a high-risk area in northeast Thailand. Int J Cancer, 59, 505-9.

Hoeben A, Landuyt B, Highley M, et al (2004). Vascular endothelial growth factor and angiogenesis. Pharmacol Rev, 56, 549-80.

Janakiram NB, Rao CV (2012). Inos-selective inhibitors for cancer prevention: promise and progress. Future Med Chem, 4, 2193-204.

Karadayi N, Kandemir NO, Yavuzer D, et al (2013) Inducible nitric oxide synthase expression in gastric adenocarcinoma: impact on lymphangiogenesis and lymphatic metastasis. Diagn Pathol, 8, 1-12.

Kerr RG, Chen Z (1995). In vivo and in vitro biosynthesis of saponins in sea cucumbers. J Nat Prod, 58, 172-76.

Kitagawa I, Kobayashi M, Hori M, et al (1989). Marine natural products. XVIII. Four lanostane-type triterpene oligoglycosides, bivittosides A, B, C, and D, from the okinawan sea cucumber bohadschia bivittata mitsukuri. Chem Pharma Bull, 37, 61-7.

Kloz T, Bloch W, Volberge C, et al (1998). Selective expression of inucible nitric oxide synthase in human prostate carcinoma. Cancer (phila), 82, 1897-903.

Kojima M, Morisaki T, Tsukahara Y, et al (1999). Nitric oxide synthase expression and nitric oxide production in human colon carcinoma tissue. J Surg Oncol, 70, 222-29.

Lechner M, Lirk P, Rieder J (2005). Inducible nitric oxide synthase (iNOS) in tumor biology: the two sides of the same coin. Semin Cancer Biol, 15, 227-89.

Li H, Huang D, Gao Z, et al (2013). Scutellarin inhibits the growth and invasion of human tongue squamous carcinoma through the inhibition of matrix metalloproteinase-2 and -9

and $\alpha v \beta 6$ integrin. Int J Oncol, 42, 1674-81.

McCawley L, Matrisian L (2000). Matrix metalloproteinases: multifunctional contributors to tumor progression. Mol Med Today, 6, 149-56.

Nabeshima K, Inoue T, Shimao Y, et al (2002). Matrix metalloproteinases in tumor invasion: role for cell migration. Pathol Int, 52, 255-64.

Niu G, wright KL, Huang M, et al (2002). Constitutive Stat 3 activity up-regulates VEGF expression and tumor angiogenesis. Oncogene, 21, 2000-08.

Pasco S, Brassart B, Ramont L, et al (2005). Control of melanoma cell invasion by type IV collagen. Cancer Detect Prev, 29, 260-66.

Plengsuriyakarn T, Viyanant V, Eursitthichai V, et al (2012). Anticancer activities against cholangiocarcinoma, toxicity and pharmacological activities of Thai medicinal plants in animal models. BMC Complement Altern Med, 12, 1-19.

Prakobwong S, Gupta SC, Kim JH, et al (2011). Curcumin suppresses proliferation and induces apoptosis in human biliary cancer cells through modulation of multiple cell signaling pathways. Carcinogen, 32, 1372-80.

Sanceau J, Truchet S, Bauvois B (2003). Matrix metalloproteinase- 9 silencing by RNA interference triggers the migratory-adhesive switch in Ewing's sarcoma cells. $J$ Biol Chem, 278, 36537-46.

Song Z-J, Gong P, Wu G-Y (2002). Relationship between the expression of iNOS, VEGF, tumor angiogenesis and gastric cancer. World J Gastroenterol, 8, 591-95.

Stonix VA, Kalinin VI, Avilov SA (1999). Toxin from sea cucumbers (Holothuroids): chemical structures, properties, taxonomic distribution, biosynthesis and evolution. $J$ Nat Toxins, 8, 235-48.

Storr SJ, Safuan S, Mitra A, et al (2012). Objective assessment of blood and lymphatic vessel invasion and association with macrophage infiltration in cutaneous melanoma. Mod Pathol, 25, 493-504.

Straume O, Jackson DG, Akslen LA (2003). Independent prognostic impact of lymphatic vessel density and presence of low-grade lymphangiogenesis in cutaneous melanoma. Clin Cancer Res, 9, 250-56.

Swana HS, Smith SD, Perrotta PL, et al (1999). Inducible nitric oxide synthase with transitional cell carcinoma of the bladder. J Urol, 161, 630-34.

Thomsen LL, Lawton FG, Knowles RG, et al (1994). Nitric oxide synthase activity in human gynecological cancer. Cancer Res, 54, 1352-54.

Thummarati P, Wijitburaphat S, Prasopthum A, et al (2012). High level of urokinase plasminogen activator contributes to cholangiocarcinoma invasion and metastasis. World $J$ Gastoenterol, 18, 244-50.

Tian F, Zhang X, Tong Y, et al (2005). PE, a new sulfated saponin from sea cucumber, exhibits anti-angiogenic and anti-tumor activities in vitro and in vivo. Cancer Biol Ther, 4, 874-82.

Tong Y, Zhang X, Tian F, et al, (2005). Philinopside A, a novel marine-derived compound possessing dual anti-angiogenenic and anti-tumor effects. Int J Cancer. 114, 843-53.

Vakkala M, Kahlos K, Lakari E, et al (2000). Inducible nitric oxide synthase expression, apoptosis, and angiogenesis in in situ and invasive breast carcinomas. Clin Cancer Res, 6, 2408-16.

Westermarck J, Kähäri V (1999). Regulation of matrix metalloproteinase expression in tumor invasion. FASEB $J$, 31, 781-92.

Xu W, Liu LZ, Loizdou M, et al (2002). The role of nitric oxide in cancer. Cell Res, 12, 311-20.

Xue Y, Chen F, Zhang D, et al (2009). Tumor-derived VEGF modulates hematopoiesis. J Angiogenes Res, 1, 1-9.

Yan L, Li L, Li Q, et al (2015). Expression of signal transducer and activator of transcription 3 and its phosphorylated form is significantly upregulated in patients with papillary thyroid cancer. Exp Ther Med, 9, 2195-201.

Ye J, Coulouris G, Zaretskaya I, et al (2012). Primer-BLAST: A tool to design target-specific primers for polymerase chain reaction. BMC Bioinformatics, 13, 134.

Yu H, Zhang S, Zhang R, et al (2009). The role of VEGF-C/D and Flt-4 in the lymphatic metastasis of early-stage invasive cervical carcinoma. J Exp Clin Canc Res, 28, 1-6. 